\title{
Technique for aortic valve annuloplasty using an intra-annular "hemispherical" frame
}

\author{
J. Scott Rankin, MD, ${ }^{\mathrm{a}, \mathrm{b}}$ L. Alan Beavan, MSE, ${ }^{\mathrm{b}}$ and William E. Cohn, $\mathrm{MD}^{\mathrm{c}}$
}

Objective: A need exists for a stable annuloplasty method for aortic valve repair. On the basis of a "hemispherical" model of aortic valve geometry, a prototype annuloplasty frame was constructed and initially tested. Specific goals were to develop a clinically applicable transaortic implant technique and to evaluate the system in isolated and intact animal preparations.

\begin{abstract}
Methods: Eight isolated porcine aortic roots were perfused from a water reservoir at a constant pressure of $100 \mathrm{~mm} \mathrm{Hg}$, and valve leak was measured by timed collection in a beaker. Baseline leak was negligible, and the 2 commissures adjacent to the right coronary leaflet were incised vertically to create severe valve insufficiency. Through a transverse aortotomy, a "hemispherical" annuloplasty frame was sutured to the aortic valve annulus with horizontal mattress sutures. The 3 posts of the frame were sutured first to the 3 subcommissural regions to align the device horizontally and vertically. The 3 frame curvatures then were sutured into the leaflet annuli using horizontal mattress "looping" sutures, supported with supra-annular pledgets. Post-repair valve leak was measured, and significance of the change was assessed with a 2-tailed paired $t$ test. One survival implant was evaluated in an intact calf.
\end{abstract}

Results: Subcommissural incision disrupted annular geometry and created a valve leak of $1400 \pm 847 \mathrm{~mL} / \mathrm{min}$ (mean \pm standard deviation). Suturing the 3-dimensional annuloplasty frame into the aortic valve annulus reestablished appropriate geometry of leaflet coaptation and restored valve competence, with a reduction in valve leak to $102 \pm 86 \mathrm{~mL} / \mathrm{min}(P=.004)$. After 6 weeks of chronic calf implantation, the frame was well healed and the native valve functioned normally.

Conclusions: Transaortic insertion of a "hemispherical" annuloplasty frame into severely disrupted and insufficient porcine aortic valves routinely and effectively restored valve competence. These data support the continued development and testing of this device as a stable method of annuloplasty during aortic valve repair. (J Thorac Cardiovasc Surg 2011;142:933-6)

Aortic valve repair is being performed more frequently for disorders causing aortic valve insufficiency and likely will have significant advantages over prosthetic valve replacement. ${ }^{1}$ At present, annular reduction usually is accomplished with commissural annuloplasty, ${ }^{2}$ a suture technique that closes the top $1 \mathrm{~cm}$ of all 3 aortic valve commissures. Although this approach is effective acutely, concern exists about the late potential for annular redilatation, and a need exists for a full annuloplasty method during aortic valve repair. To provide a fixed geometric annuloplasty of

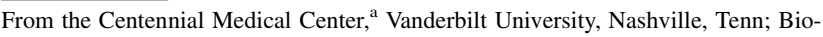
Stable Science and Engineering, ${ }^{\mathrm{b}}$ Austin, Tex; and Texas Heart Institute, ${ }^{\mathrm{c}}$ Houston, Tex

This study was funded by BioStable Science and Engineering Inc, Austin, Texas. Disclosures: J.S.R. is a consultant for BioStable Science and Engineering Inc, and Chief Medical Officer of HAART, Inc. L.A.B. is employed by both companies, and all authors have investment positions with these companies. All authors had complete scientific freedom in experimental design, data acquisition, data analysis, and publication.

Received for publication May 19, 2010; revisions received Dec 23, 2010; accepted for publication Jan 21, 2011; available ahead of print March 7, 2011

Address for reprints: J. Scott Rankin, MD, 320 Lynnwood Blvd, Nashville, TN 37205 (E-mail: jsrankinmd@cs.com).

$0022-5223 / \$ 36.00$

Copyright (C) 2011 by The American Association for Thoracic Surgery

doi:10.1016/j.jtcvs.2011.01.039
}

the entire aortic valve annulus, a 3-dimensional frame has been developed, based on the geometry of 3 leaflet "hemispheres" nested within a cylindric aorta. ${ }^{3,4}$ Coupled with leaflet reconstruction procedures, such as "plication" and autologous pericardial augmentation, ${ }^{5-9}$ this device could provide a highly applicable and stable approach to aortic valve repair. Initial testing of the device by suturing into isolated aortic valve annuli from below the valve was encouraging. ${ }^{4}$ The purpose of this study was to develop a clinically applicable transaortic technique for frame insertion and to test the efficacy of the method in isolated and intact animal aortic roots.

\section{MATERIALS AND METHODS}

The "hemispherical" model of aortic valve geometry and the design of the annuloplasty frame have been described in detail. ${ }^{3,4}$ Briefly, the valve leaflets were represented as 3 hemispheres nested within a cylindric aorta. Because the junction of the leaflets and aorta could be used to represent the aortic valve annulus, the intersection of the 3-dimensional spherical equations for the leaflets and the cylindric equation for the aorta produced a 3-pointed crown-shaped representation of the aortic valve annulus. On the basis of this model, a computerized milling machine was programmed with the 3-dimensional coordinates of the annulus for each diameter, and 1-piece frames were machined from tubes of titanium over a variety of sizes. From the model, the frame posts were vertical, and the 
post heights were equal to frame radius at each diameter. Dacron sewing rings were attached to the frames, allowing the devices to be sutured to the aortic valve annulus (Figure 1, $A$ ).

The testing preparation was developed elsewhere. ${ }^{4}$ Eight fresh-frozen isolated porcine aortic roots were cannulated and pressurized from a water reservoir at a height above the aorta equivalent to $100 \mathrm{~mm} \mathrm{Hg}$ pressure. Leakage of the aortic valve was measured by 30 -second collection of regurgitant water into a $500-\mathrm{mL}$ beaker positioned under the preparation. After baseline competence was confirmed, vertical incisions were made progressively into the 2 commissures on either side of the right coronary leaflet until a regurgitant volume of approximately $1000 \mathrm{~mL} / \mathrm{min}$ was obtained (Figure 1, B). Timed collection of the regurgitant volume was measured. A transverse incision then was made in the root of the aorta. The freeedge length of the right coronary leaflet was measured, and a frame diameter was chosen with a circumference of twice the leaflet free-edge length. ${ }^{4}$

By using Cabrol-like stitches, ${ }^{2}$ the 3 posts of the frame first were sutured to the 3 commissural areas with the frame on a holder outside the aorta (Figure 2, A). In this way, frame posts were initially fixed under the commissures, and the frames were oriented circumferentially to the valve. The vertical level of each post in each subcommissural area was gauged to bring the bottom of the frame into apposition with the bottom of the leaflets. Insertion of the posts too high in the subcommissural region distorted the bottom of the leaflets and caused prolapse. Aligning the posts first, both vertically and horizontally, brought the frame into initial proper orientation within the valve and prevented skewed insertion with resultant leaflet distortion.

The frame then was detached from the holder and lowered into position below the valve leaflets, while the post sutures were left untied. With the frame in position and taking the stitches in 1 bite, 2 to 3 additional horizontal mattress sutures (depending on the size of the valve) were placed in the annulus of each leaflet, being careful to loop the frame. Initially, bites were taken in the Dacron sewing ring, but subsequently this was eliminated and the frame just looped (Figure 2, B). Looping the frame seemed to strengthen the quality of insertion. Although the Dacron covering was not used for suturing, it stabilized the suture grip and facilitated frame endothelialization. Each group of 2 to 3 leaflet sutures were placed deeply into the annulus from below, with the sutures emerging above the valve in the sinus of Valsalva. The mattress sutures then were tied in 1 step over custom-designed small-dimension Dacron pledgets. A final view from above the valve after frame annuloplasty is shown in Figure 2, $C$. With this procedure, the frame posts were buried back into the subcommissural region, and no part of the frame, pledgets, or sutures contacted the delicate leaflet tissue. The aortotomy then was closed with a running suture, the valve was repressurized at $100 \mathrm{~mm} \mathrm{Hg}$ (Figure 2, D), and a repeat measurement of regurgitant volume was obtained. Changes in valve leak from the disrupted to post-annuloplasty state were assessed with a 2-tailed paired $t$ test.

To test the implant technique in vivo, a $92-\mathrm{kg}$ calf was placed on cardiopulmonary bypass with standard cardioplegia and humanquality survival surgery. The aortic root was opened transversely, and the valve was sized to a $21-\mathrm{mm}$ diameter frame, which was inserted with the technique described. The device was the same as used in the isolated root studies, except the posts were flared outward slightly to facilitate "burying" them into the subcommissural area. The frame seated well, and the aorta was closed. After separation from bypass, an epicardial echocardiogram showed good leaflet motion and no leak, and the animal recovered uneventfully. At 6 weeks postoperatively, a repeat echocardiogram was obtained before euthanasia and examination of the gross specimen. This experiment was performed after animal protocol approval and according to the animal experimentation guidelines of the Texas Heart Institute.

\section{RESULTS}

Commissural incision disrupted leaflet geometry and produced severe aortic valve regurgitation, averaging $1400 \mathrm{~mL} / \mathrm{min}$ (Table 1). Annuloplasty frame insertion from above the valve and through the aorta was considerably more difficult, compared with direct intra-annular suturing from below. ${ }^{4}$ As the procedure described in this article was refined, however, the technical ease was facilitated. Frame annuloplasty significantly reduced the leak (Table 1; $P=.004$ ), and a learning curve was evident. As experience was gained with the method described, a progressively more precise orientation of the frame was obtained to the valve, and residual leak decreased to low levels with technical improvement (Table 1). Insertion of a frame into the intact calf produced no valve difficulties, and the postoperative period was uncomplicated. Echocardiogram at 6 weeks again showed good leaflet motion and no leak. Pictures of the gross specimen from above and below the valve are shown in Figure 3.
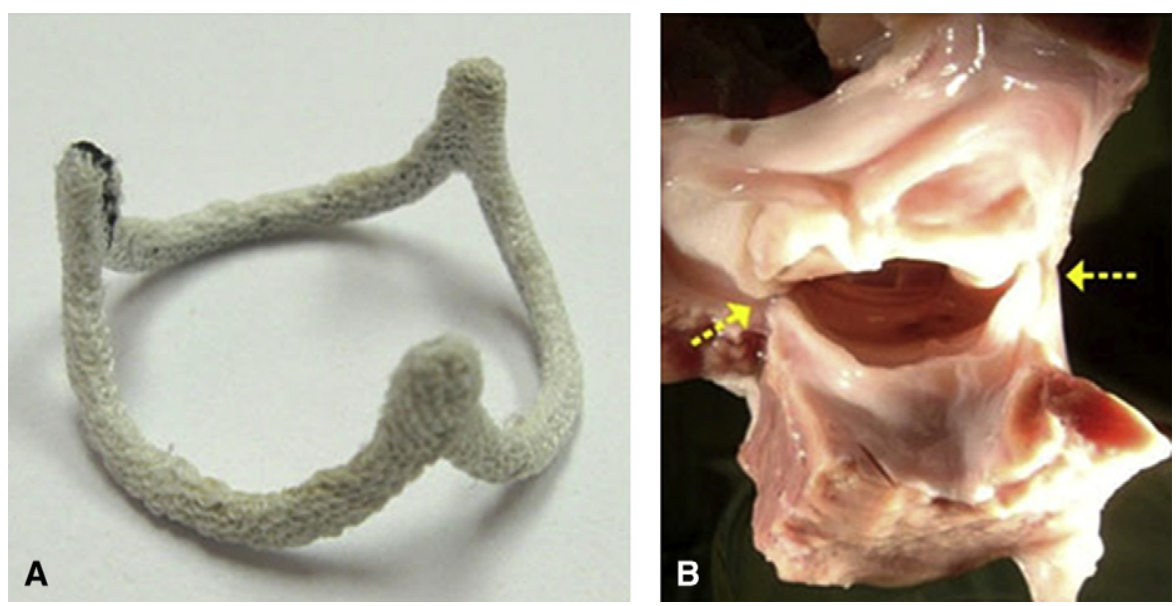

FIGURE 1. A, Photograph of a prototype "hemispherical" annuloplasty frame. B, Vertical incisions in the commissures on either side of the right coronary cusp (yellow arrows). See text for details. 

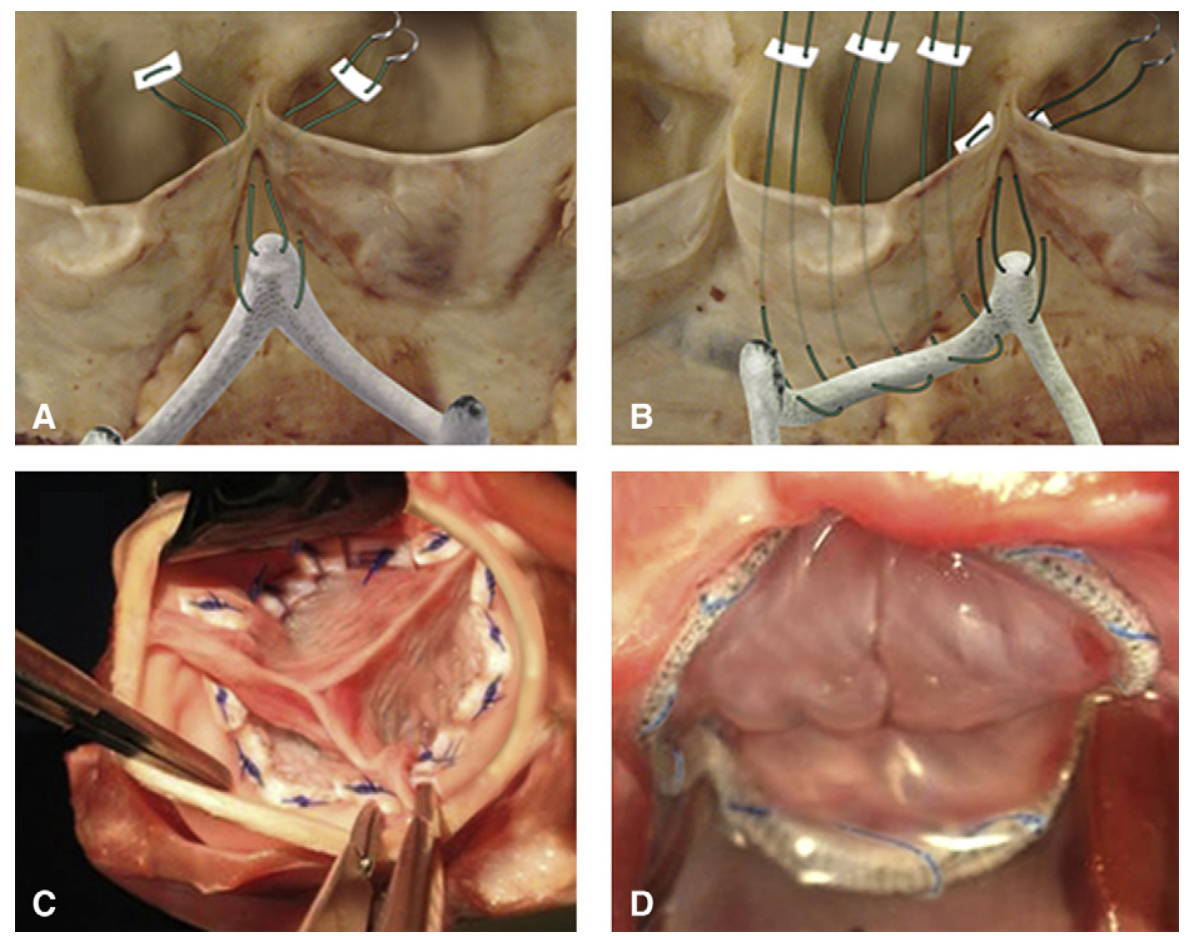

FIGURE 2. A, Three frame posts were first sutured to the subcommissural regions to orient the device vertically and horizontally. B, After insertion, looping horizontal mattress sutures were placed around the body of the frame and then vertically through the valve annulus. After all sutures were placed, they were tied over small custom-designed Dacron pledgets that were narrow enough not to extend out to the leaflet tissue. Initially, small bites of Dacron were taken, but the method seemed to work better (and proceed faster) with just looping of the frame, eliminating the Dacron bites. C, View of the implanted valve from above with most of the aorta cut away. Leaflets are symmetric with good coaptation and vertical position. D, View of the valve from below after pressurization showing good frame position and cusp coaptation after restoration of leaflet geometry.

\section{DISCUSSION}

Many methods of aortic valve annuloplasty have been devised, but they often tended to distort leaflet geometry and even could increase leak. In 1966, Cabrol and associates ${ }^{2}$ reported subcommissural annuloplasty, basically suturing closed the top $1 \mathrm{~cm}$ of each commissure with horizontal mattress sutures. This technique not only reduced annular diameter but also altered root geometry by raising the leaflets to a more vertical coaptation plane. In cases with mild to moderate root pathology and no leaflet prolapse,

\section{TABLE 1. Effects of annuloplasty on valve leak}

\begin{tabular}{lcc}
\hline Study & Incised leak $(\mathbf{m L})$ & Repaired leak $(\mathbf{m L})$ \\
\hline 1 & 1200 & 292 \\
2 & 1000 & 125 \\
3 & 1100 & 125 \\
4 & 2400 & 100 \\
5 & 1200 & 70 \\
6 & 3000 & 40 \\
7 & 700 & 50 \\
8 & 600 & 16 \\
Mean \pm SD & $1400 \pm 847$ & $102 \pm 86$ \\
\hline
\end{tabular}

$S D$, Standard deviation. With commissural incision, a gross leak was produced and insertion of the annuloplasty frame effectively returned valve competence toward normal. A learning curve was evident, and residual leak seemed to improve as insertion technique was refined (see text for methods). commissural annuloplasty performed well. Subsequently, commissural annuloplasty was combined with free-edge "plication" to correct leaflet prolapse, and it became possible to repair a greater number of disorders causing aortic insufficiency. ${ }^{5-9}$ However, achieving a stable long-term aortic valve annuloplasty remains a significant concern, because suture annuloplasties of other valves have had significant late failure rates.

Permanent fixation of annular geometry during mitral valve repair usually is accomplished with ring annuloplasty. However, development of a similar "annuloplasty ring" for the aortic valve has been hampered by the fact that the aortic annulus is not a "ring,"3 and the complex 3-dimensional geometry has been difficult to understand. Therefore, an effort was made to define annular geometry in the human cadaver laboratory, ${ }^{3}$ and that work produced a "hemispherical" model of the aortic valve, in which the junction of the leaflets and aorta could be represented mathematically. From those studies, a prototype annuloplasty frame was developed (Figure 1), and feasibility was tested successfully as a repair device in incompetent aortic valves. ${ }^{4}$ However, development and testing of a clinically applicable method of frame insertion were lacking, and these were the goals of this study.

Transaortic insertion of the frame beneath the valve was more difficult than expected, and the method described in 

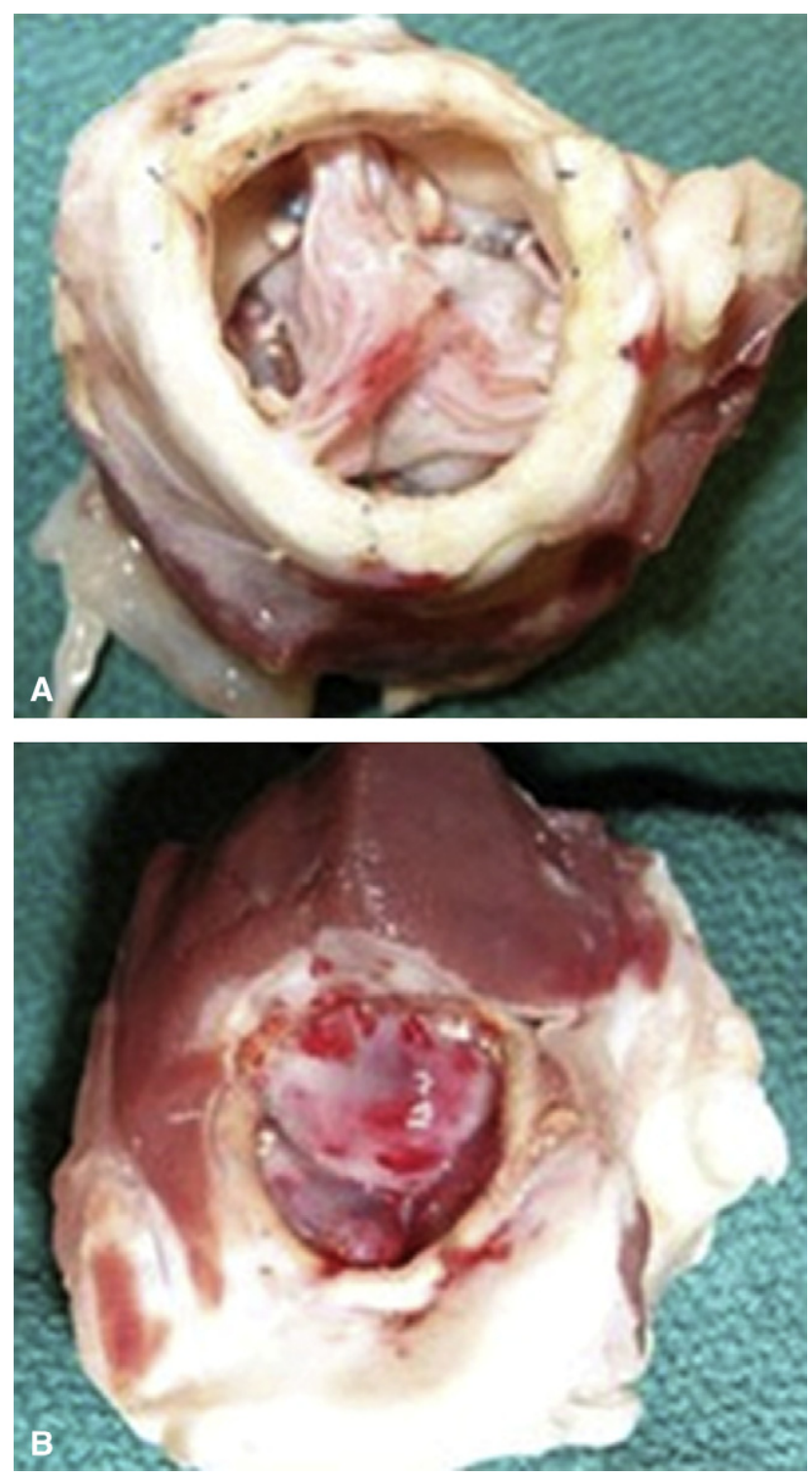

FIGURE 3. A, View of the explanted calf aortic valve from above at 6 weeks after frame insertion with the technique described. The leaflets were symmetric with good coaptation and vertical position. B, View of the valve from below showing good frame position and endothelialization/healing of the device with competent leaflets.

this article was gradually developed. With this approach, frame placement became easier, and disrupted valves could be realigned to good leaflet coaptation and valve competence. Moreover, the intra-annular frames were "tucked- away" in the subcommissural space and beneath the leaflets where they would not interfere with leaflet function or produce leaflet abrasion in the chronic setting. The result seemed to approximate the native leaflets mounted on a stent, producing what one might consider an "autologous" biologic valve (Figures 2 and 3). These findings will now lead to testing in chronic animal systems, using the insertion method developed in this study and assessing safety and efficacy in the biologic setting. This study should be construed only as a "proof of concept" analysis, because the model of subcommissural incision is artificial at best and does not resemble the clinical pathologic anatomy of aortic insufficiency. Future chronic animal studies together with analysis of human valve geometry will likely produce further technical or design modifications, but it is now evident that this general approach may be worthwhile.

\section{CONCLUSIONS}

It should be stressed that this frame would comprise only 1 component of aortic valve repair: a stable long-term method of annuloplasty. Preexisting leaflet prolapse or associated abnormalities would need to be addressed with leaflet plication or other procedures ${ }^{5-9}$ to optimize leaflet "effective height" and coaptation area. However, coupled with appropriate leaflet procedures, and including a variety of asymmetrical and bicuspid frames, clinical application of this type of annuloplasty could make aortic valve repair a routine clinical procedure with stable longterm results.

\section{References}

1. Aicher D, Fries R, Rodionycheva S, et al. Aortic valve repair leads to a low incidence of valve-related complications. Eur J Cardiothorac Surg. 2010;37:127-32.

2. Cabrol C, Cabrol A, Guiraudon G, et al. Le traitement de l'insuffisance aortique par l'annuloplstie aortique. Arch Mal Coeur Vaiss. 1966;59:1305-12.

3. Rankin JS, Dalley AF II, Crooke PS, et al. A 'hemispherical' model of aortic valvar geometry. J Heart Valve Dis. 2008;17:179-86.

4. Rankin JS. An intra-annular 'hemispherical' annuloplasty frame for aortic valve repair. J Heart Valve Dis. 2010;19:97-103.

5. Aicher D, Langer F, Adam O, et al. Cusp repair in aortic valve reconstruction: does the technique affect stability? J Thorac Cardiovasc Surg. 2007;134:1533-9.

6. Schäfers H-J. Reconstruction of the bicuspid aortic valve. Op Tech Thorac Cardiovasc Surg. 2007;12:2-13.

7. Khoury G, Vanoverschelde JL, Glineur D, et al. Repair of aortic valve prolapse: experience with 44 patients. Eur J Cardiothorac Surg. 2004;26:628-33.

8. Chiappini B, Pouleur A-C, Noirhomme P, et al. Repair of trileaflet aortic valve prolapse: mid-term outcome in patients with normal aortic root morphology. Interact Cardiovasc Thorac Surg. 2007;6:56-9.

9. Shäfers H-J, Aicher D, Langer F. Correction of leaflet prolapse in valve preserving aortic replacement: pushing the limits? Ann Thorac Surg. 2002;74:1762-74. 